\title{
Performance of the CMS electromagnetic calorimeter at the LHC and role in the hunt for the Higgs boson
}

\author{
Riccardo Paramatti* \\ On behalf of the CMS Collaboration \\ INFN Rome \\ E-mail: riccardo.paramattieromal.infn.it
}

\begin{abstract}
The Electromagnetic Calorimeter (ECAL) of the Compact Muon Solenoid (CMS) experiment at the LHC is a hermetic, fine grained, homogeneous calorimeter, comprising 75,848 lead tungstate $\left(\mathrm{PbWO}_{4}\right)$ scintillating crystals, located inside the CMS superconducting solenoidal magnet. The scintillation light is detected by avalanche photodiodes (APDs) in the barrel section and by vacuum phototriodes (VPTs) in the two endcap sections. A silicon/lead preshower detector is installed in front of the endcaps in order to improve $\gamma / \pi^{0}$ discrimination. Precise calibration of the ECAL detector is required. This includes inter-calibration, to account for the differing response of channels, and calibration of the energy scale. The performance obtained during the first LHC physics runs in 2010 and 2011 is presented and the role of the ECAL in the hunt for the Higgs boson, through the two-photon decay mode, is discussed.
\end{abstract}

36th International Conference on High Energy Physics,

July 4-11, 2012

Melbourne, Australia

\footnotetext{
* Speaker.
} 


\section{Introduction}

The Compact Muon Solenoid (CMS) experiment [1] at the LHC is designed to investigate the electroweak symmetry breaking mechanism through the search for the Higgs boson and to search for new physics at the unexplored $\mathrm{TeV}$ scale. One of the most sensitive channels in the search for a low mass Higgs boson is the two-photon decay mode where the excellent energy resolution of the electromagnetic calorimeter (ECAL) [2] plays a fundamental role. The natural width of the (low mass) Standard Model Higgs boson is negligible compared to the width due to the instrumental resolution and the signature of the $\gamma \gamma$ channel is a narrow peak in the invariant mass distribution of the two photons over a large QCD background. This report presents the performance of the CMS ECAL, with particular emphasis on the aspects relevant for the Higgs boson search in the two-photon decay channel. The stability, channel calibration, and energy resolution are discussed. The results presented use proton-proton collision data from LHC operation in 2010 and 2011 at a centre-of-mass energy of $7 \mathrm{TeV}$ with integrated luminosities of $36 \mathrm{pb}^{-1}$ and $4.98 \mathrm{fb}^{-1}$ respectively.

\section{The electromagnetic calorimeter of CMS}

The CMS ECAL is a compact and homogeneous calorimeter made of 75848 lead-tungstate $\left(\mathrm{PbWO}_{4}\right)$ crystals, arranged in a quasi-projective geometry. The barrel region (EB) covers a pseudorapidity range up to $|\eta|=1.48$ while the two endcaps (EE) extend up to $|\eta|<3.0$. The scintillation light is readout with avalanche photodiodes (APDs) in EB and with vacuum phototriodes (VPTs) in EE. The transverse size of the crystals is very close to the $\mathrm{PbWO}_{4}$ Molière radius of $2.2 \mathrm{~cm}$ while the crystal depth is about 26 and 25 radiation lengths $\left(X_{0}=0.89 \mathrm{~cm}\right)$ respectively in EB and EE, providing nearly complete longitudinal containment of the electromagnetic shower for electrons and photons with energies up to $50 \mathrm{GeV}$ and beyond. A preshower detector (ES), based on lead absorbers equipped with silicon strip sensors, is placed in front of the endcap crystals.

The performance of the calorimeter has been extensively tested with electron beams: the stochastic and the electronic noise contributions to the energy resolution of ECAL have been shown to match the design requirements (2.8\% and $120 \mathrm{MeV}$ respectively with a $3 \times 3 \mathrm{~EB}$ crystal matrix). The overall resolution was proven excellent and well below $1 \%$ at high energies, with an irreducible constant term of about $0.3 \%$ for particles impinging on the centre of the crystals [3]. These results have been used to tune the MC simulation. The in-situ additional contributions to the energy resolution, mainly affecting the constant term, are caused by residual miscalibrations of the channel-to-channel response within ECAL and by channel response changes with time, due to radiation damage of the crystals and phototriodes response loss. The energy resolution is further degraded for electrons and photons that interact in the tracker material in front of ECAL.

\section{ECAL Monitoring and Calibration}

The main effect on the ECAL crystals of ionising and non-ionising radiation at the LHC is a wavelength-dependent loss of crystal transparency without change to the scintillation mechanism. A secondary effect of the radiation is that the VPT response decreases to a plateau with accumulated photocathode charge. In order to measure and correct for response change during LHC operation, 


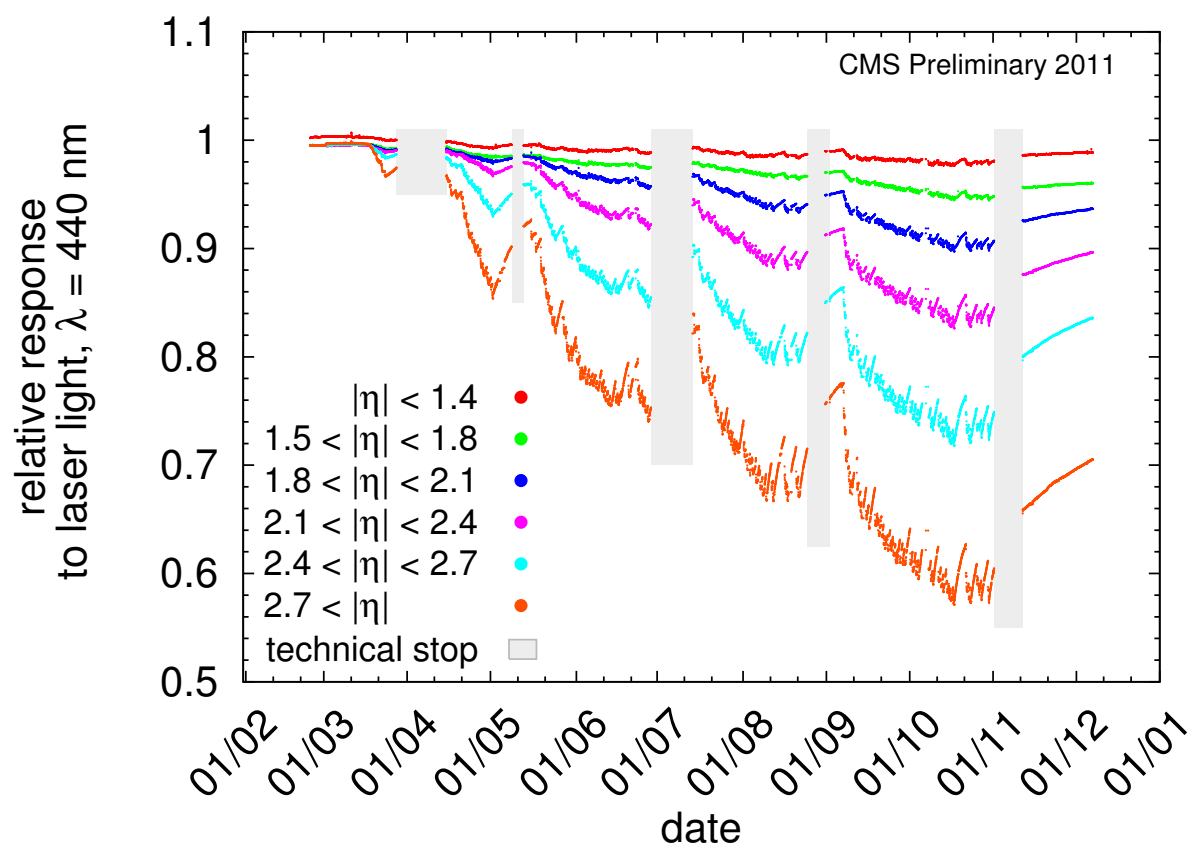

Figure 1: Relative response variation measured by the laser monitoring system during the 2011, normalised to the beginning of 2011. The shaded areas correspond to technical stops of LHC which are without monitoring data while the November heavy ion running corresponds to a crystal recovery period. The response is averaged over the pseudorapidity ranges listed in the legend.

the ECAL is equipped with a dedicated laser monitoring system[4]. The evolution of the ECAL response to laser data in 2011 is shown in Figure 1 as a function of time. An average value is shown for each of six pseudorapidity ranges. The data are normalised to the measurements at the start of 2011. Periods without colliding beams are shaded. The laser signals drop exponentially during periods of LHC operation, reaching a saturation level which depends on the dose-rate (note that the dose-rate increased in the different periods due to the increasing LHC luminosity). The observed losses are consistent with expectations and reach $3 \%$ in EB, about $15 \%$ at the end of the CMS acceptance region for e/ $\gamma(|\eta|<2.5)$ and up to $40 \%$ at the end of EE. The validity of these corrections is continuously monitored by following the time stability of $\pi^{0} / \eta \rightarrow \gamma \gamma$ and $Z \rightarrow e^{+} e^{-}$ invariant masses and the $E / p$ ratio of $W \rightarrow e v$ electrons (see section 4).

The spread of the channel-to-channel response is mainly due to the the crystal light yield variation in EB, about $15 \%$ at construction, and to the gain spread of the photodetectors in EE, of about $25 \%$. Uncorrected, this spread affects the constant term of the energy resolution of ECAL with little dilution as the fraction of energy deposited in the central crystal of the electromagnetic shower is up to $70 \%$. Calibration procedures, such as laboratory measurements of the crystal light yield, test-beam with electrons, calibration with cosmic muons, have been adopted during the construction and commissioning of ECAL [5]. Since the beginning of LHC $p-p$ running several in-situ calibration strategies have been used [6]:

- $\phi$-symmetry inter-calibration, to provide fast inter-calibration by exploiting the invariance around the beam axis of energy flow in minimum bias events. 
- $\pi^{0}$ and $\eta$ calibration, using the photon pairs selected as $\pi^{0} / \eta \rightarrow \gamma \gamma$ candidates.

- isolated electrons from $W \rightarrow e v$ and $Z \rightarrow e^{+} e^{-}$decays, comparing the energy measured in ECAL to the track momentum measured in the silicon tracker.

The inter-calibration precision of each method has been estimated by comparing the individual results, and cross-checking against precalibration constants. Results obtained in the 2011 run are shown in Fig. 2 as a function of pseudorapidity for EB (left) and EE (right). While the $\phi$-symmetry and $\pi^{0} / \eta$ precisions are systematically limited, the isolated electron calibration is still statistically limited and is expected to become the main calibration channel in the subsequent years.
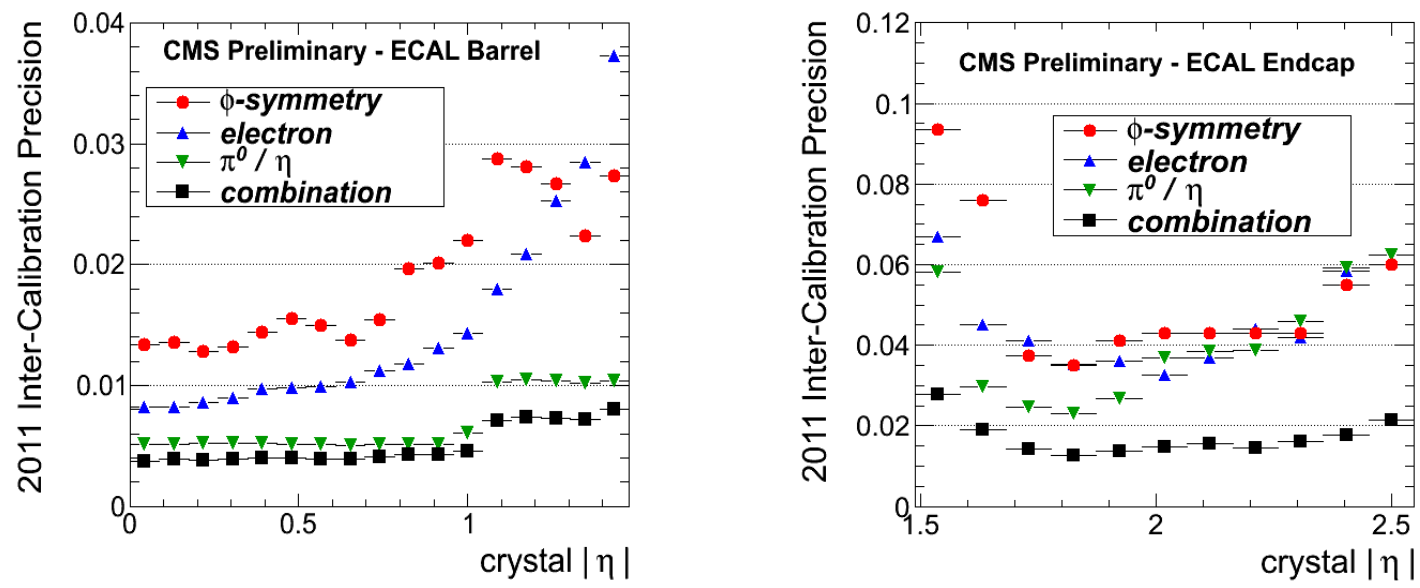

Figure 2: The inter-calibration precision obtained in 2011 using $\phi$ symmetry, the $E / p$ ratio with electrons, $\pi^{0} / \eta$ decays, and the resultant precision for the combination of the methods, in EB (left) and EE (right).

\section{The reconstruction of electron/photon energy in ECAL}

The estimation of the electron and photon energy in ECAL involves the collection of the energy deposited by the electromagnetic shower over several crystals. At the test beam the optimal energy resolution is obtained by summing the energy deposited in fixed arrays of crystals, as $3 \times 3$ or $5 \times 5$, around the crystal with the most energy. In-situ, the combined action of the strong magnetic field and the presence of tracker material requires the use of dynamic clustering algorithms to recover clusters of energy deposits due to electron bremsstrahlung or photon conversions[7]. The energy of an e/ $\gamma$ candidate is estimated by the following sum:

$$
E_{e, \gamma}=F_{e, \gamma} \cdot\left[G \cdot \sum_{i} S_{i}(t) \cdot C_{i} \cdot A_{i}+E_{E S}\right]
$$

where $A_{i}$ is the reconstructed signal amplitude in ADC counts, $S_{i}(t)$ the time response variation obtained by the laser monitoring data, $C_{i}$ the channel-to-channel intercalibration and $G$ the ADCto-GeV conversion factor which fixes the energy scale of the EB and EE separately. For EE clusters the preshower energy $E_{E S}$ is added and finally a particle-dependent correction factor $F_{e / \gamma}$ is applied 
to compensate for unclustered energy and energy not reaching the calorimeter. This factor has a value between $0 \%$ and $10 \%$ and is strongly related to the amount of material in front of ECAL.

The energy measured by ECAL for isolated electrons from $W$ and $Z$ decays is used to monitor the stability of the response and resolution. The overall relative response stability throughout the 2011 is at the $0.1 \%$ level in EB (as shown in Fig. 3-left) and the 0.3\% in EE. The stability of ECAL resolution in 2011, monitored with the relative instrumental width of the $\mathrm{Z}$ invariant mass peak, is excellent in EB while a slight degradation is observed in EE (Fig. 3-right) partially due to the increased number of collisions per beam crossing during the year. The instrumental contribution to the $Z$ width $\left(\sigma_{C B}\right)$ is extracted from a fit to the invariant mass distribution of a Breit-Wigner convoluted with a Crystal-Ball response function [8].
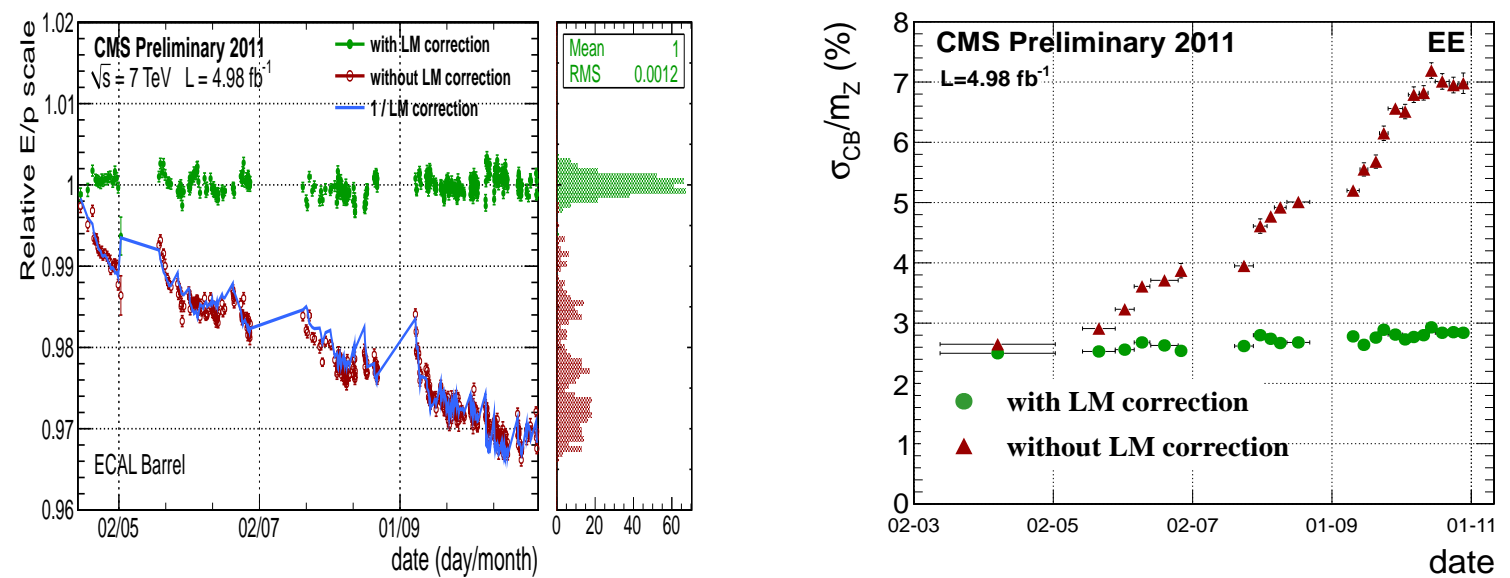

Figure 3: Stability of the ECAL response during 2011 with isolated electrons in the ECAL barrel (left) and the stability of the ECAL resolution during 2011 with $Z \rightarrow e^{+} e^{-}$events in the ECAL endcaps (right). Red (green) points show data before (after) the laser monitoring corrections.

\section{ECAL energy and $H \rightarrow \gamma \gamma$ invariant mass resolution.}

The in-situ energy resolution for electrons is measured using $Z \rightarrow e^{+} e^{-}$events with an unbinned maximum likelihood fit to the reconstructed invariant mass distribution where the energy resolution of the electrons is a free parameter as a function of $|\eta|$. The procedure is applied to both 2011 Data and MC simulation and the results are shown in Fig. 4. In the CMS simulation, the ECAL energy resolution (noise, stochastic and constant contributions) has been tuned with Test Beam measurements. In addition a gaussian spread for the single channel response, corresponding to the estimated residual miscalibration as shown in Fig. 2, is applied. However response instabilities are not yet simulated; moreover imperfections in the simulation of the material in front of ECAL can also contribute to the observed difference between Data and MC.

The search for the Higgs Boson in the two-photon decay channel relies on the energy resolution and the position measurement within ECAL of the reconstructed photons. In order to precisely simulate the response to photons, the constant term of the energy resolution in the MC is tuned in different regions of ECAL and for the different electromagnetic shower shapes to match 

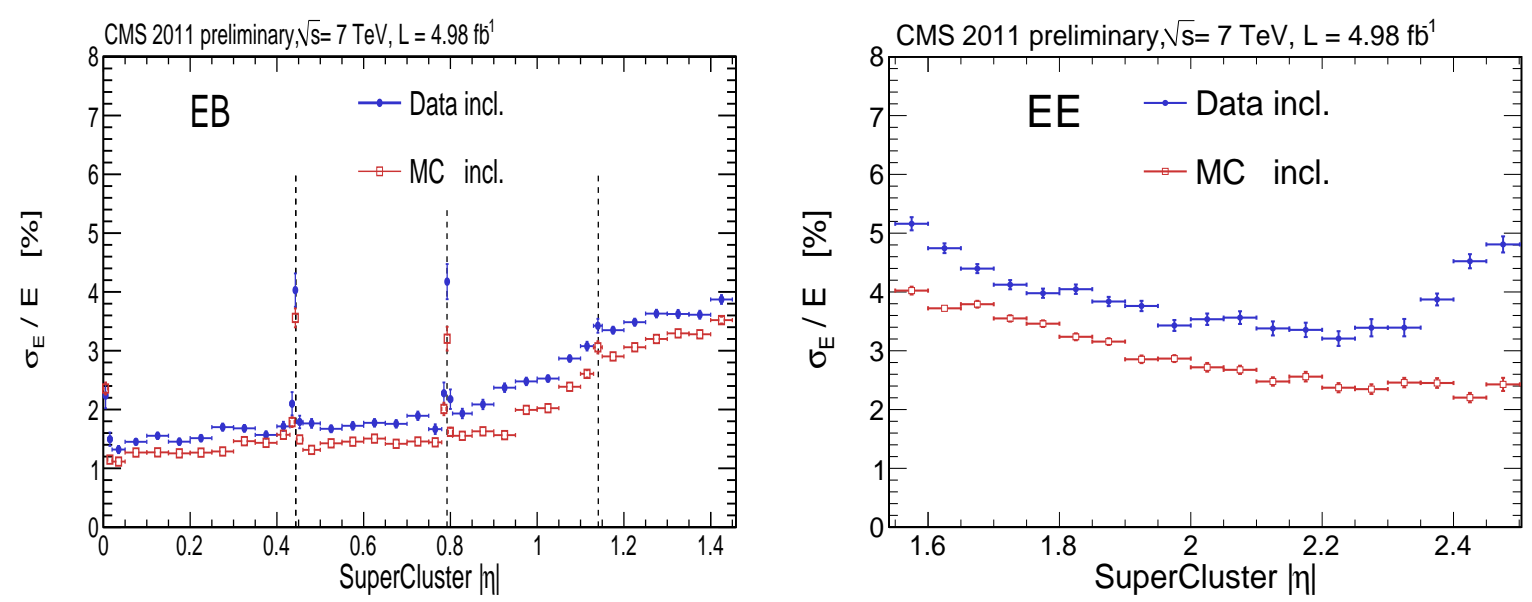

Figure 4: Relative energy resolution as a function of absolute pseudo-rapidity for $Z \rightarrow e^{+} e^{-}$electrons from Data and MC for EB (left) and EE (right). The vertical dashed lines are the module boundaries in EB.

the observed resolution in $Z \rightarrow e^{+} e^{-}$data, with electrons reconstructed as photons, except for the vertex. After this MC tuning using data, the relative invariant mass width of simulated $H \rightarrow \gamma \gamma$ events (expressed as FWHM/2.35) is $1.17 \%$ and $0.90 \%$ respectively for the inclusive sample and the golden channel, where both photons are in EB and the shower shapes are compatible with unconverted photon. These results take into account also the additional contribution to the invariant mass resolution coming from the uncertainty of the measured angle between the two photons. The latter contribution, given the excellent precision of the ECAL shower position reconstruction [9], is however negligible with respect to the photon energy resolution term if the diphoton vertex is reconstructed to better than $1 \mathrm{~cm}$ precision by using information from the tracks associated with the vertex. This precision is obtained when the collision vertex is correctly identified (for about $80 \%$ of $H \rightarrow \gamma \gamma$ events [10]).

\section{Conclusions}

The excellent performance of the CMS electromagnetic calorimeter during the LHC 2010 and 2011 physics runs at $7 \mathrm{TeV}$ played an important role in the search for the Higgs Boson in the twophoton decay channel and in the recent observation of a new boson at a mass of $125 \mathrm{GeV}$ by the CMS Collaboration [11]. The stability of the ECAL response and resolution as a function of time in the high radiation environment has been measured with isolated electrons from the $\mathrm{W}$ and $\mathrm{Z}$ boson decays. The electron energy resolution, measured from the invariant mass peak width of $Z \rightarrow e^{+} e^{-}$ events collected in 2011, varies between $1.5 \%$ in the central part of the barrel to about $4 \%$ in the endcaps. Further progress, to improve the precision of channel intercalibration, the understanding of the effects of the tracker material and the tuning of clustering algorithms, is expected in the near future. 


\section{References}

[1] Chatrchyan S et al., The CMS experiment at the CERN LHC', JINST 3 (2008) S08004

[2] The CMS Collaboration, The Electromagnetic Calorimeter Project: Technical Design Report, CERN/LHCC 1997, CMS TDR 4

[3] Adzic P et al., Energy resolution of the barrel of the CMS Electromagnetic Calorimeter JINST 2 (2007) P04004

[4] Anfreville M et al., Laser monitoring system for the CMS lead tungstate crystal calorimeter, Nucl. Instr. and Meth. A 594 (2008) 292-320.

[5] Adzic P et al., Intercalibration of the barrel electromagnetic calorimeter of the CMS experiment at start-up, JINST 3 (2008) P10007

[6] CMS Collaboration, Electromagnetic calorimeter calibration with $7 \mathrm{TeV}$ data, CMS PAS-EGM-2010-003 (2010)

[7] CMS Collaboration, CMS Physics Technical Design Report, Volume I, CERN-LHCC-2006-021

[8] Skwarnicki T, Ph.D Thesis, DESY F31-86-02(1986), Appendix E, and references therein.

[9] CMS Collaboration, ECAL Detector Performance, 2011 Data, CERN-CMS-DP-2012-007 (2012)

[10] Chatrchyan S et al., Search for the standard model Higgs boson decaying into two photons in pp collisions at $\sqrt{s}=7 \mathrm{TeV}$, Phys. Lett. B 710 (2012) 403

[11] Chatrchyan S et al., Observation of a new boson at a mass of $125 \mathrm{GeV}$ with the CMS experiment at the LHC, Phys.Lett. B 716 (2012) 30-61 